\title{
Au Nanoparticle Formation from Amorphous Au/Si Multilayer
}

\author{
Masami Aono1, Takashi Ueda', Hiroshi Abe ${ }^{1 *}$, Shintaro Kobayashi², Katsuhiko Inaba² \\ ${ }^{1}$ Department of Materials Science and Engineering, National Defense Academy, Yokosuka, Japan \\ ${ }^{2}$ Application Laboratory, Rigaku Co., Akishima, Tokyo, Japan \\ Email: ${ }^{*}$ ab@nda.ac.jp
}

Received 17 July 2014; revised 17 August 2014; accepted 16 September 2014

Copyright (C) 2014 by authors and Scientific Research Publishing Inc.

This work is licensed under the Creative Commons Attribution International License (CC BY).

http://creativecommons.org/licenses/by/4.0/

(c) (i) Open Access

\section{Abstract}

By direct observations of transmission electron microscopy (TEM), irreversible morphological transformations of as-deposited amorphous $\mathrm{Au} / \mathrm{Si}$ multilayer (a-Au/a-Si) were observed on heating. The well arrayed sequence of the multilayer changed to zigzag layered structure at $478 \mathrm{~K}$ $\left(=T_{\text {zig }}\right)$. Finally, the zigzag structure transformed to Au nanoparticles at $508 \mathrm{~K}$. The distribution of the Au nanoparticles was random within the thin film. In situ X-ray diffraction during heating can clarify partial crystallization $\mathrm{Si}$ (c-Si) in the multilayer at $450 \mathrm{~K}\left(=T_{\mathrm{MIC}}^{\mathrm{ML}}\right)$, which corresponds to metal induced crystallization (MIC) from amorphous Si (a-Si) accompanying by Au diffusion. On further heating, a-Au started to crystallize at around $480 \mathrm{~K}\left(=T_{\mathrm{c}}\right)$ and gradually grew up to $3.2 \mathrm{~nm}$ in radius, although the volume of $\mathrm{c}-\mathrm{Si}$ was almost constant. Continuous heating caused crystal $\mathrm{Au}$ (c-Au) melting into liquid AuSi ( $\ell$-AuSi) at $600 \mathrm{~K}\left(=T_{\mathrm{m}}^{\mathrm{NP}}\right)$, which was lower than bulk eutectic temperature $\left(T_{\mathrm{E}}^{\mathrm{B}}=636 \pm 5 \mathrm{~K}\right)$. Due to the AuSi eutectic effect, reversible phase transition between liquid and solid occurred once temperature is larger than $T_{\mathrm{m}}^{\mathrm{NP}}$. Proportionally to the maximum temperatures at each cycles $(673,873$ and $1073 \mathrm{~K})$, both $T_{\mathrm{m}}^{\mathrm{NP}}$ and Au crystallization temperature approaches to $T_{\mathrm{E}}^{\mathrm{B}}$. Using a thermodynamic theory of the nanoparticle formation in the eutectic system, the relationship between $T_{\mathrm{m}}^{\mathrm{NP}}$ and the nanoparticle size is explained.

\section{Keywords}

Amorphous Au/Si Multilayer, Au Nanoparticle, Low Eutectic Point, Metal Induced Crystallization, Irreversible Morphological Transformation, Reversible $\ell$-AuSi-c-Au Nanoparticle Phase Transition

\footnotetext{
${ }^{*}$ Corresponding author.
}

How to cite this paper: Aono, M., Ueda, T., Abe, H., Kobayashi, S. and Inaba, K. (2014) Au Nanoparticle Formation from Amorphous Au/Si Multilayer. Journal of Crystallization Process and Technology, 4, 193-205. 


\section{Introduction}

In metal silicides, metal-induced crystallization (MIC) [1]-[5] in thin films is well known to be a fundamental phenomenon relating to low eutectic point. Rather than viewing this phenomenon from a solid-phase reaction perspective, studies from the diffusion process view point are necessary due to the fact that electric screening weakens the covalent bonds of the amorphous $\mathrm{Si}$ (a-Si). Diffusion process in amorphous is a key to interpret the various phenomena in Au-Si system and apply it into a novel process for further functional materials.

The MIC process is classified into two groups: 1) eutectic alloy formation and 2) metallic compound assisted process. The former suggests that metal diffusion into a-Si is the main reason of MIC determined from the formation process of eutectics. By in situ observation of microstructure using electron microscopy [6], it was found that $\mathrm{Au}$ particles appear on the surface of a-Si at $573 \mathrm{~K}$. Diffuse zone was also found between crystalline Au (c-Au) particles and the surrounding a-Si. Moreover, diffusion of Au into Si substrates was examined by X-ray reflectivity [7]. Surface sensitive diffusion is separated into Fickian-/non-Fickian-type, which is directly related to the surface stability. The latter described in 2) is characterized by a variety of crystal structures of the AuSi particles, which are induced in hydrogenated amorphous Si (a-Si:H) [8] and c-Au/a-Si thin film [9]. The metastable Au-silicides $\left(\mathrm{Au}_{2} \mathrm{Si}[10], \mathrm{Au}_{5} \mathrm{Si}_{2}, \mathrm{Au}_{7} \mathrm{Si}\right.$, and so on) decomposed at higher temperature.

The surface nucleation on the substrate was investigated with respect to the eutectic phenomenon of $\mathrm{Au}_{1-x} \mathrm{Si}_{x}$ [11]-[13]. A significant fact about the super cooling of c-Au nanoparticles on the Si (111) surface has been clarified by in situ observation of X-ray scattering and $a b$ initio molecular dynamics [13]. It is understood that a nanoeutectic phenomenon is that the crystal nucleation is suppressed by a lateral order at interface. In the simulation box, a pentagonal cluster appears to be reconstructed on the interface.

Relating to crystal nucleation, irreversible morphological transformation occurred by the self-assembled mechanism [14]. The mono disperse Au nanoparticles were obtained by thermal decomposition of the multilayer films. a- $\mathrm{Au}_{25} \mathrm{Si}_{75} / \mathrm{a}-\mathrm{Si}$ multilayer was deposited on c-Si substrate. The stacking sequence is a-Si/a- $\mathrm{Au}_{25} \mathrm{Si}_{75} / \mathrm{a}-$ $\mathrm{Si} / \mathrm{a}-\mathrm{Au}_{25} \mathrm{Si}_{75} / \mathrm{a}-\mathrm{Si} / \ldots$ with varying the a- $\mathrm{Au}_{25} \mathrm{Si}_{75}$ thickness as 10, 25, 50 and $100 \mathrm{~nm}$, where the a-Si thickness is fixed at $100 \mathrm{~nm}$. As a different morphology in mono layer, spiral patterns of Au nanoparticles were formed in addition to the conventional dendritic phases [15]. The geometric array was generated in one $\mathrm{Au}_{1-\chi} \mathrm{Si}_{x}$ thin film on $\mathrm{Si}$ substrate, whose thickness is $25 \mathrm{~nm}$. On the other hand, cyclic $4.2 \mathrm{~nm}$ a-Si and $0.8 \mathrm{~nm}$ a-Au multilayer showed different irreversible transformations such as nanoparticles after annealing [16]. The thin film consists of 60 layers. Both the as-deposited and annealed samples were investigated in transmission electron microscope (TEM) observations, X-ray diffraction (XRD) and Raman scattering spectroscopy. The randomly located Au nanoparticle was observed after annealing. Also, the morphological changes were seen in Au/Ge multilayer, known as low eutectic point [17]. The size of c-Au particles increases with increasing Au layer thickness.

For industrial applications, nano-processing using binary eutectic alloys is utilized widely in a various kinds of scientific fields. For instance, well-arrayed Au nanoparticle enables control of coherent localized optical fields, which can be applied to chemical and biological sensors [18]. Surface plasmon driven by dark or bright resonance is very sensitive to the nanoscale fabrication. Artificially engineered structures are applied into plasmonic nano-systems assembled with chemical and biological molecules. In other plasmon technology, the Au nanoparticles were assembled into a solar cell. Periodic arrangement of the Au nanoparticles in the devices can cause plasmon polariton modes on the surface of the Au nanoparticles. Low eutectic point in $\mathrm{Au}_{1-\chi} \mathrm{Si}_{x}$ can fabricate easily on the a-Si film [19], a thin film of organic electroluminescence diode [20] and ZnO diode [21]. Other representative applications in nano-engineering, write-once optical disk [22] and rewritable data storage [23] were proposed.

In this study, we performed in situ observations of TEM and XRD to clarify the formation mechanism of cAu nanoparticle from a-Au/a-Si multilayer. Based on the diffusion-limited model, Au aggregations are explained. Repulsive interaction in the vicinity of eutectic point supports to form the Au nanoparticle. At each thermal cycles, melting point of c-Au nanoparticle $\left(T_{\mathrm{m}}^{\mathrm{NP}}\right)$ depends on the nanoparticle size of c-Au as predicted theoretically.

\section{Experimental Details}

The (012) surface of $\mathrm{Al}_{2} \mathrm{O}_{3}$ was selected for the substrate. Samples were prepared using Si (High Purity Chemicals Co., 99.9995\%) and Au (High Purity Chemicals Co., 99.95\% up). In the ultrahigh vacuum system $\left(10^{-8}-\right.$ $\left.10^{-6} \mathrm{~Pa}\right)$, Si and Au were deposited selectively. Au $0.8 \mathrm{~nm} / \mathrm{Si} 4.2 \mathrm{~nm}, 60$ layers were deposited by molecular 
beam epitaxy (MBE) method (INFICON XTC). The stacking sequence is $\mathrm{Al}_{2} \mathrm{O}_{3} / \mathrm{Si} / \mathrm{Au} / \mathrm{Si} / \mathrm{Au}$... Si/Au. The deposition rate was estimated to be $0.05 \mathrm{~nm} / \mathrm{s}$. Each thickness is represented to be $t_{\mathrm{Au}}$ and $t_{\mathrm{S}}$, respectively. Their volume ratio, $V_{\mathrm{Au}} / V_{\mathrm{Si}}$, is proportional to the thickness one. Therefore, the volume of $\mathrm{Si}$ is $84 \%$ of the whole volume (Table 1). On the other hand, the volume ratio in the thin film is simply converted into concentration. Si concentration, $x$, of the as-deposited sample $\left(\mathrm{Au}_{1-\chi} \mathrm{Si}_{x}\right)$ is expressed by,

$$
x:(1-x)=\frac{m_{\mathrm{Si}}}{M_{\mathrm{Si}}}: \frac{m_{\mathrm{Au}}}{M_{\mathrm{Au}}}=\frac{\rho_{\mathrm{Si}} V_{\mathrm{Si}}}{M_{\mathrm{Si}}}: \frac{\rho_{\mathrm{Au}} V_{\mathrm{Au}}}{M_{\mathrm{Au}}}=\frac{\rho_{\mathrm{Si}} t_{\mathrm{Si}}}{M_{\mathrm{Si}}}: \frac{\rho_{\mathrm{Au}} t_{\mathrm{Au}}}{M_{\mathrm{Au}}} .
$$

Here, $M_{\mathrm{Au}}$ and $M_{\mathrm{Si}}$ are atomic weight of Au and Si. $\rho_{\mathrm{Au}}$ and $\rho_{\mathrm{Si}}$ are density of a-Au and a-Si, respectively. In previous studies, density of a-Au was roughly estimated to be around $9.1 \mathrm{~g} / \mathrm{cm}^{3}$ in [24], while a-Si was evaluated to be $2.33 \mathrm{~g} / \mathrm{cm}^{3}$ in [25]. In $\mathrm{Au}_{1-x} \mathrm{Si}_{x}$, Si composition of the thin film used in this study is equivalent to $x=0.904$. By fixing at $t_{\mathrm{Au}}=0.8 \mathrm{~nm}, t_{\mathrm{Si}}$, which contributes to eutectic composition $\left(x_{\mathrm{E}}=0.186\right)$, becomes $0.102 \mathrm{~nm}$ (Table 1). If the whole volume of $\mathrm{Au}(16 \%)$ could melt above melting point in the eutectic $\mathrm{Au}_{1-x} \mathrm{Si}_{x}\left(x=x_{\mathrm{E}}\right)$, additional $2.0 \% V_{\mathrm{Si}}$ is required to form the liquid at $x_{\mathrm{E}}$.

At high temperature, direct morphological observations were carried out using a field emitted transmission electron microscopy (TEM, JEOL JEM-2100F, $200 \mathrm{kV}$ ) with energy dispersive X-ray spectroscopy (EDS) and charge coupled detector (CCD). Temperature was monitored using Pt-Pt 13\% Rh (Type-R). The thin films for cross-sectional TEM characterization were prepared by focused ion beam (FIB, Hitachi FB-2100) with Ga ion. The temperature dependence of electrical conductivity of the samples was measured using a four-point probe in dry $\mathrm{N}_{2}$ flow.

In situ XRD was performed using $9 \mathrm{~kW}$ rotating anode type X-ray generator (SmartLab, Rigaku Co.). $45 \mathrm{kV}$ and $200 \mathrm{~mA}$ were set for measurements. $\mathrm{Cu} \mathrm{K} \alpha$ radiation $(\lambda=0.1542 \mathrm{~nm})$ was selected and collimated using parabolic multilayer mirror. Furthermore, the beam was focused by a converting optical tool (CBO-f, Rigaku Co.): the beam size was estimated to be $400 \mu \mathrm{m}$ in diameter. High temperature furnace (DHS900, Anton Paar Co.) was attached on a $\theta-\theta$ goniometer. X-ray window of the furnace was carbon hemisphere, whose thickness is $0.7 \mathrm{~mm}$. During in situ observations, dry $\mathrm{N}_{2}$ gas was flowing at $50 \mathrm{cc} / \mathrm{min}$ after vacuum in order to prevent oxidation. Both heating and cooling rate was $10 \mathrm{~K} / \mathrm{min}$. The experimental conditions in continuous four thermal cycling were programmed as shown in Figure 1. Each holding temperatures $\left(T_{\max }\right)$ were 473, 673, 873 and 1073 K. 2D detector (PILATUS 100K/R) was used for rapid scan (10 sec/one 2D pattern). The thin film used in in situ XRD was synthesized at the same condition as the as-deposited sample used in the TEM and conductivity measurements

\section{Results and Discussion}

\subsection{Morphological Transformations by Direct TEM Observation}

TEM images both of as-deposited and annealed thin films are shown in Figure 2. The annealed temperature was $873 \mathrm{~K}$. In the pictures of the cross section of the multilayer, the dark parts reveal the Au layers. In the as-deposited thin film, the well-stacked Au and Si layers were observed (Figure 2(a)). By Fourier transform (FT) of atomic arrangement in Figure 2(a), the as-deposited thin film was found to be amorphous. On the other hand, the layered structure completely disappeared after annealing (Figure 2(b)). Instead, Au nanoparticles existed in the thin film, where the nanoparticles randomly locate inside Si. FT of the Au nanoparticles in Figure 2(b) suggests that the nanoparticles crystallized from amorphous by annealing. Thus, irreversible morphological transformation occurred accompanying by crystallization of the Au nanoparticles (c-Au).

Table 1. Thickness of the multilayer and volume fractions of $\mathrm{Au}$ and $\mathrm{Si}$ at each state.

\begin{tabular}{|c|c|c|c|c|c|c|}
\hline \multicolumn{3}{|c|}{ As-deposited } & \multirow{2}{*}{\multicolumn{3}{|c|}{$\begin{array}{r}T>T_{\mathrm{m}} \\
V(\%)\end{array}$}} & \multirow{3}{*}{$\begin{array}{r}T<T \\
V(\% \\
16\end{array}$} \\
\hline & $t(\mathrm{~nm})$ & $V(\%)$ & & & & \\
\hline \multirow[t]{2}{*}{$\mathrm{a}-\mathrm{Au}$} & 0.8 & 16 & $\mathrm{Au}$ in $\ell$-AuSi & 16 & $\mathrm{c}-\mathrm{Au}$ & \\
\hline & & & Si in $\ell$-AuSi & $\sim 2.0$ & & \\
\hline \multirow[t]{2}{*}{$\mathrm{a}-\mathrm{Si}$} & 4.2 & 84 & $\mathrm{a}-\mathrm{Si}$ & $\sim 64.8$ & $\mathrm{a}-\mathrm{Si}$ & $\sim 66.8$ \\
\hline & & & c-Si & $\sim 17.2$ & c-Si & $\sim 17.2$ \\
\hline
\end{tabular}




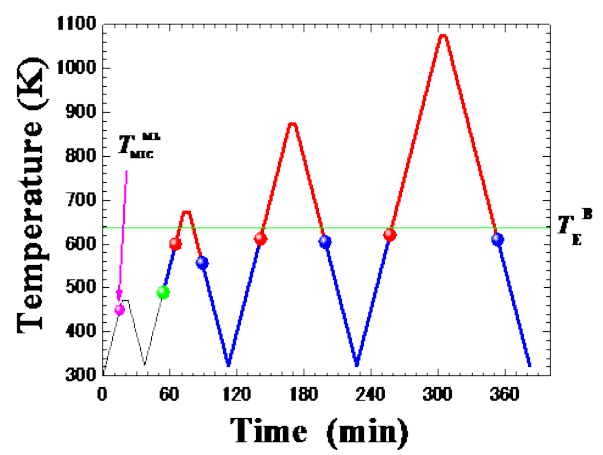

Figure 1. Thermal history of in situ observation of X-ray diffraction. Melting point and crystallization temperature at each thermal cycles are expressed by red blue closed circles, respectively. Both heating and cooling rate was 10 $\mathrm{K} / \mathrm{min}$. Bulk eutectic point $\left(T_{\mathrm{E}}^{\mathrm{B}}\right)$ is indicated by green line. Thick blue and red lines represent crystal- and liquid-Au, respectively. Amorphous Au is expressed by thin black line. $T_{\mathrm{MIC}}^{\mathrm{ML}}$ is temperature of metal-induced crystallization of a-Si in the multilayer.
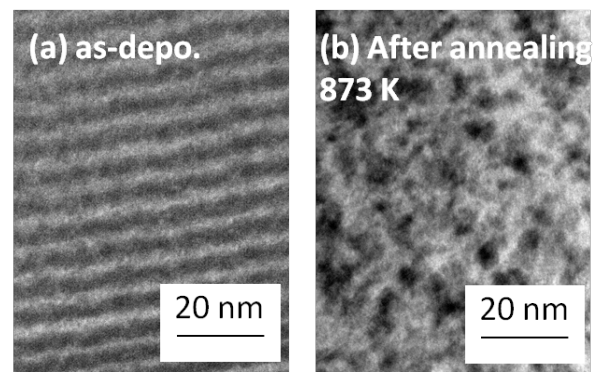

Figure 2. Cross-sectional TEM images of (a) the as deposited a-Au/a-Si and (b) the annealed thin film having the c-Au nanoparticles. The annealing temperature is $873 \mathrm{~K}$.

In situ observation using TEM is indispensable to clarify the intermediate process of the above morphological transformation from the multilayer to Au nanoparticles in real space. Upon heating, the layered structure of Au was changing gradually. At $393 \mathrm{~K}$ as shown in Figure 3(a), roughness at the boundary between a-Au and a-Si occurred partially on the atomic scale. The modulation of the boundaries in the multilayer implies that Au diffusion might be activated thermally below the MIC temperature $\left(T_{\mathrm{MIC}}^{\mathrm{ML}}=450 \mathrm{~K}\right)$. By further heating above $T_{\mathrm{MIC}}^{\mathrm{ML}}$, zigzag patterns were observed at $478 \mathrm{~K}\left(=T_{\mathrm{zig}}\right)$ with losing the flatness and smoothness (Figure 3(b)). We suppose that, above $T_{\mathrm{MIC}}^{\mathrm{ML}}$, much Au diffusion is promoted on the mesoscopic scale. Consequently, the initial layered structure of a-Au might be modified into the zigzag one by mass transfer of Au. At $508 \mathrm{~K}$, the metastable zigzag pattern in the thin film vanished drastically (Figure 3(c)). Simultaneously, some dynamic fluctuations of Au nanoparticles were probed by the in situ TEM observation. The nano-heterogeneity is characterized by its ambiguous boundary and dynamic motion. The nearly static zigzag pattern at $478 \mathrm{~K}$ and slow dynamic nano-heterogeneity at $508 \mathrm{~K}$ (second order) are regarded as a precursor phenomenon of the Au nanoparticle formation.

\subsection{Crystal/Amorphous Estimations by XRD}

Both the as-deposited Si and Au layers is an amorphous state, which is determined by XRD (Figure 4(a)). In fact, there is no sharp Bragg spot in the 2D X-ray diffraction except for those from the substrate. The weak spots are from the substrate with different wavelengths such as $\mathrm{Cu} K \beta_{1}(\lambda=0.1392 \mathrm{~nm})$ and $\mathrm{W} \operatorname{L} \alpha_{1}(\lambda=0.1476 \mathrm{~nm})$ due to filament of $\mathrm{X}$-ray generator. Judging from no Debye rings, the as-deposited multilayer in $\mathrm{Au}_{1-x} \mathrm{Si}_{x}$ consists only of a-Au and a-Si. In the sample preparations, Au atoms cannot form crystal in the multilayer during deposition, since $t_{\mathrm{Au}}(=0.8 \mathrm{~nm})$ might be too thin to achieve the critical size for Au crystal nucleation. It is confirmed from a previous study using bilayered c-Au/a-Si $\left(t_{\mathrm{Au}}=30 \mathrm{~nm}\right)$ [6], that the Au thickness is sufficient for the crystal nucleation of Au.

On the other hand, after cycle $4\left(T_{\max }=1073 \mathrm{~K}\right)$, Au crystallization was distinguished by the Debye rings on 


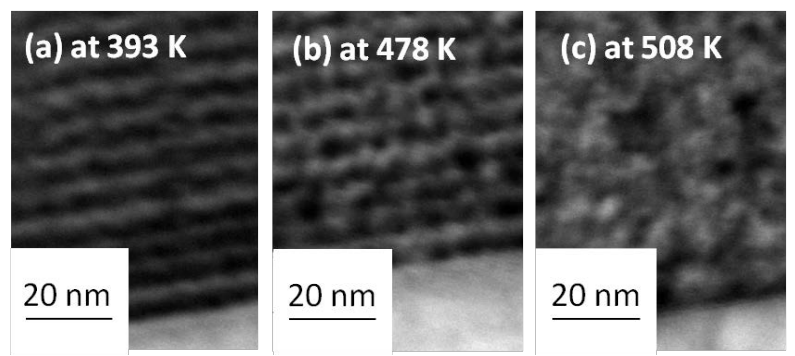

Figure 3. In situ observations by TEM at (a) $393 \mathrm{~K}$, (b) $478 \mathrm{~K}$ and (c) $508 \mathrm{~K}$. As a precursor phenomenon to Au nanoparticles, zigzag pattern is formed at around $480 \mathrm{~K}$, whose temperature is equivalent to the Au crystallization temperature determined by XRD. Au nanoparticle appeared at $510 \mathrm{~K}$.
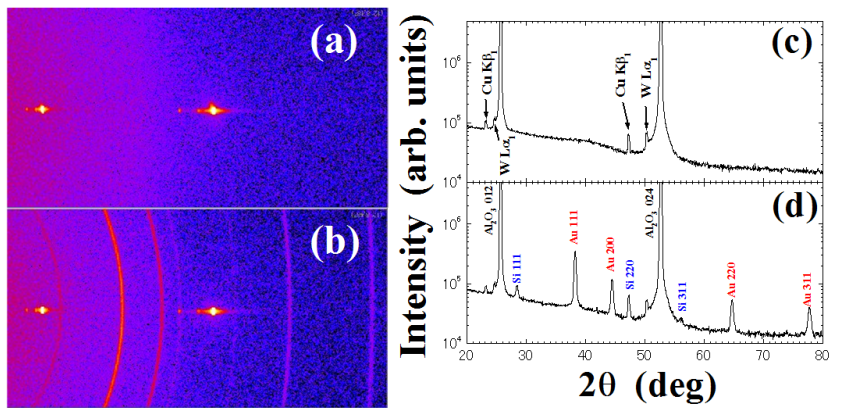

Figure 4. 2D X-ray diffraction patterns (a) before thermal cycle and (b) after cycle 4. The 2D data are reduced into 1D patterns (c) before thermal cycle and (d) after the 4th cycle.

the XRD pattern (Figure 4(b)). From the peak widths, the average size of c-Au nanoparticles was estimated at room temperature and was determined to be $3.0 \mathrm{~nm}$ in radius. The ideal Debye rings of the Bragg reflections in Figure 4(b) suggest that the Au nanoparticles have no orientational order in the thin film. Thus, after annealing, the c-Au nanoparticles were observed at random sites by TEM and random crystal orientations by XRD. Therefore, XRD data does not contradict with the TEM observations relating to the morphological transformation from multilayer to Au nanoparticles. To compare the Au crystal with Si one, the observed 2D data were reduced into 1D (Figure 4(c) and Figure 4(d)). Moreover, after cycle 4, metastable Au silicides such as $\mathrm{Au}_{2} \mathrm{Si}_{1} \mathrm{Au}_{5} \mathrm{Si}_{2}$ and $\mathrm{Au}_{7} \mathrm{Si}$ [8]-[10] were not seen at room temperature (Figure 4(b)). Metastable silicides were bit seen during in situ XRD observations. By Williamson-Hall method [26], local root-mean-square strain of c-Au was analyzed using the thermal cycling sample. From the peak widths of Bragg reflections in c-Au (Figure 4(d)), strain of the c-Au nanoparticles is negligibly small.

\subsection{Au Nanoparticle Formation by in Situ XRD}

Figures 5(a)-(d) show the respective integrated intensities of Si 111 Bragg reflection at each thermal cycle. At cycle 1, a-Si crystallized partially and irreversibly at around $450 \mathrm{~K}\left(=T_{\mathrm{MIC}}^{\mathrm{ML}}\right.$ ) (Figure 5(a)). This is regarded as typical MIC, but no Au crystallization was detected. Once the MIC process occurred, the volume of c-Si was almost constant over all thermal cycles (cycles 1 - 4). The irreversible Si crystallization can be interpreted as frozen Si atoms, which are unstably captured in the multilayer, whereas the as-deposited a-Si is partially stabilized by crystallization. After cycle 4, XRD in Figure 4(b) and Figure 4(d) reveal that the Bragg intensities of $\mathrm{c}$-Au are larger than those of c-Si in spite of $t_{\mathrm{Au}}<t_{\mathrm{Si}}$. For quantitative analysis of each crystal volumes, we estimate the crystal volume ratio by X-ray kinematical theory [27]. Generally, the scattering volume, $V$, in XRD is proportional to integrated intensities of Bragg reflections. In the case of polycrystal, the relation is given by,

$$
I_{\mathrm{hkl}} \propto\left|F_{\mathrm{hkl}}\right|^{2} \mathrm{e}^{-2 \mathrm{M}} \cdot \mathrm{LP} \cdot m_{\mathrm{hkl}} \cdot V .
$$

$F_{\text {hkl }}$ is the structure factor and $\mathrm{e}^{-2 \mathrm{M}}$ is the Debye-Waller factor as thermal dumping function. LP is the Lorentz-polarization factor. $m_{\mathrm{hkl}}$ is the multiplicity factor for polycrystalline. Here, we ignore the extinction effect, since multiple scattering is not enhanced in the nanoparticles. Also, X-ray absorption of c-Au is negligible 


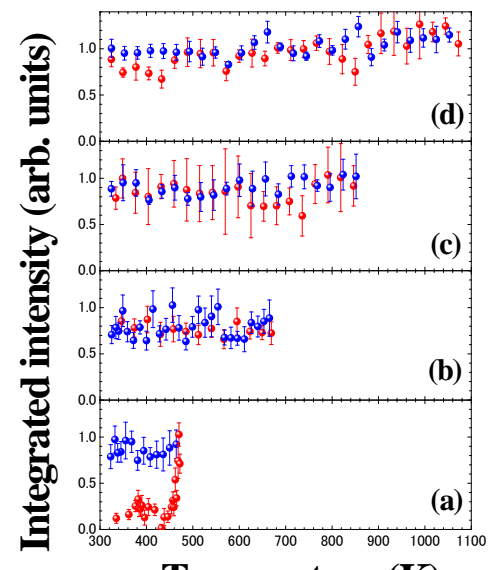

Temperature (K)

Figure 5. Integrated intensities of Si 111 Bragg reflection at (a) cycle 1, (b) cycle 2, (c) cycle 3 and (d) cycle 4. Heating and cooling data are represented by red and blue closed circles, respectively.

due to quite small nanoparticles. When the observed integrated intensities of Bragg reflections are normalized using Equation (2), the substantial volumes ratio of $V^{\mathrm{Si}}$ (c-Si) to $V^{\mathrm{Au}}$ (c-Au) is evaluated to be 1.07. If all a-Au crystallize, $V^{\mathrm{Au}}$ of c-Au becomes $16 \%$. Using the crystal volume ratio, the volume of c-Si is calculated to be $17.2 \%$ (Table 1). The rest of Si (66.8\%) still remains frozen as the amorphous state even after cycle 4. Hence, a-Si might be stable trivially in the thin film. In addition to small amount of c-Si on the MIC process, lower $T_{\mathrm{MIC}}^{\mathrm{ML}}$ $(=450 \mathrm{~K})$ was observed in this study. Previously, on heating $(3 \mathrm{~K} / \mathrm{min})$, the MIC occurred at around $483 \mathrm{~K}$ with decreasing Bragg intensity of c-Au in the thin film (30 nm of c-Au on a $200 \mathrm{~nm}$ thick a-Si) [6]. Moreover, large grained c-Si was obtained in the study. Therefore, the partial and irreversible MIC at cycle 1 in this study is entirely different from the conventional MIC process. Here, we emphasize that an initial Au state (a-Au or c-Au) influences extensively on the MIC process in a-Si.

On heating the film at cycle $2\left(T_{\max }=673 \mathrm{~K}\right.$ ), a-Au started to crystallize at around $480 \mathrm{~K}\left(=T_{\mathrm{c}}\right.$ ). Figure 6 (a) shows temperature dependence of intensity of Au 111 Bragg reflection. The crystallization temperature of Au coincides with $T_{\text {zig. }}$. In real space, zigzag patterns of Au layers in Figure 3(b) were formed at the same temperature as a-Au started to crystallize. A significant point is that Au nucleation process has some relation with the morphological changes. From the mass transfer of Au, which is explained by shape change from flat stripe to zigzag, Au diffusion might assist the crystal nucleation. The intensity of Au 111 Bragg reflection gradually increased with increasing temperature. The previous study [16] suggests that the morphological transformation from the layered a-Au to the c-Au nanoparticles takes place irreversibly. In contrast to the MIC in a-Si, it is emphasized that the whole volume of a-Au can transform to c-Au at around $560 \mathrm{~K}$. On deposition process of $\mathrm{Au}$, the confinement in the thin Au layer $\left(t_{\mathrm{Au}}=0.8 \mathrm{~nm}\right)$ below the critical size for crystal nucleation enforces the atomic arrangement to form unstable amorphous, which is far from an equilibrium state. Consequently, all a-Au can transform to crystal, since the driving force from a-Au to c-Au is large enough. Further heating at cycle 2 caused the eutectic $\mathrm{Au}_{1-\chi} \mathrm{Si}_{x}$ to melt. Above $570 \mathrm{~K}$, the intensity of c-Au decreased and disappeared completely at $600 \mathrm{~K}\left(=T_{\mathrm{m}}^{\mathrm{NP}}\right)$, although the Bragg intensities of c-Si were almost constant. Thus, it is interpreted that liquid AuSi ( $\ell$-AuSi) was formed with a mass transfer from a-Si (Table 1). Here, the bulk eutectic point $\left(T_{\mathrm{E}}^{\mathrm{B}}\right)$ in $\mathrm{Au}_{1-x} \mathrm{Si}_{x}$ is $636 \mathrm{~K}$. In the same manner with low $T_{\mathrm{MIC}}^{\mathrm{ML}}$, the thin film has a tendency of low $T_{\mathrm{m}}^{\mathrm{NP}}$. On another approach, temperature dependence of full width at half maximum (FWHM) of Au 111 Bragg reflection is calculated by profile fitting using the asymmetric pseudo-Voigt function (Figure 6(b)). Remarkably, FWHM at the early stage of crystallization became large reflecting on small nuclei from a-Au. Accompanying with coarsening up to the c-Au nanoparticles, FWHM of the Bragg reflection became smaller. At the temperature range from 540 $\mathrm{K}$ up to $600 \mathrm{~K}$, constant FWHM implies that the nanoparticle size of c-Au was suppressed at $2.6 \mathrm{~nm}$ in radius $\left(r^{\mathrm{obs}}\right)$, which is determined by the conventional Scherrer equation [28].

\subsection{Au Diffusion Process at the Early Stage of Au Crystallization}

Corresponding to the early stage of Au crystallization and the morphological change including diffusion process, 


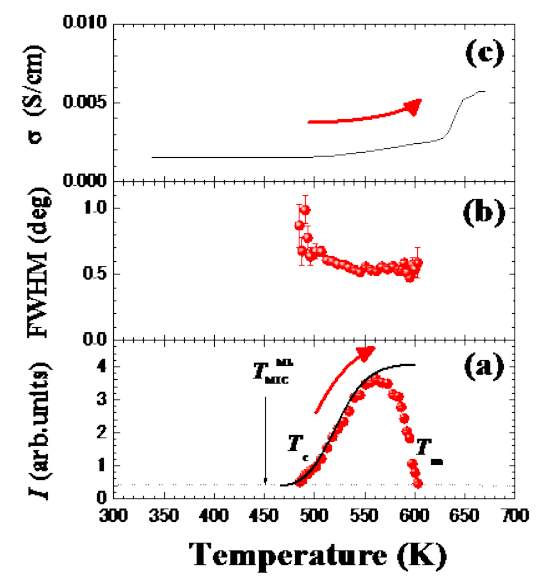

Figure 6. Heating process of (a) intensity, (b) FWHM of Au 111 Bragg reflection at the 2nd heating and (c) electrical conductivity, $\sigma$. Solid curve reveals the calculated values by the diffusion-driven nucleation model.

electrical conductivity $(\sigma)$ was measured separately using other as-deposited sample at the same heating rate (10 $\mathrm{K} / \mathrm{min}$ ) in Figure 6(c). At around $T_{\mathrm{c}}$ of c-Au, gradient of the conductivity as a function of temperature varied a little. Since $\sigma$ is expressed by mobility of species having charge, it is adequate to consider thermally induced local diffusion of Au atoms above $T_{\mathrm{c}}\left(\sim T_{\mathrm{zig}}\right)$. The abrupt increase of $\sigma$ above $T_{\mathrm{m}}^{\mathrm{NP}}$ might be originated from free-Au migration in the liquid state. In order to interpret the irreversible morphological transfer and the slow crystal growth process of Au, we assume the process by introducing the conventional "diffusion-limited model" and "interface-limited model". Just below Au crystallization temperature, Au diffusion occurs on atomic scale in amorphous multilayer. By the TEM observations, Au diffusion-driven zigzag structure supports the Au mass transfer. At the early stage of crystallization, Au atoms aggregate each other and crystal nanoparticle is formed. At the late stage of crystallization, interface-limited model is effective once size of nanoparticle exceeds the critical size. However, repulsive interaction near the eutectic point suppress the further growth of Au nanoparticle.

We focus the early stage of Au diffusion process. The local diffusion is described by slow dynamics has less geometrical constraint, that is, shape flexibility, compared with the interface-limited process. Then, we introduce the diffusion-assisted nucleation model [29] [30], which is provided by,

$$
\frac{\mathrm{d} P}{\mathrm{~d} t}=K_{n} c^{2} \exp \left\{-\frac{\Delta G(n, c)}{k_{\mathrm{B}} T}\right\},
$$

where $k_{\mathrm{B}}$ is the Boltzmann constant and $\Delta G$ is the free energy of an $n$-monomer embryo. $P$ is the size distribution function, which is represented by $n$-monomer embryo. $c$ is the monomer concentration. In the Smoluchowski expression, $K_{n}$ is approximately obtained by,

$$
K_{n}=4 \pi a n^{1 / 3} D .
$$

Here, $D$ is diffusion coefficient for monomers and $a$ is radius of the monomer. In the case of a-Au/a-Si system above $T_{\mathrm{c}}, D$ in Equation (4) corresponds to Au atomic diffusion into a-Si. Experimentally, the activation energy of Au atomic diffusion inside a-Si was obtained in the previous study [31]. The effective radius of the embryo, $r$, is expressed as $a n^{1 / 3}$ considering spherical aggregation of $n$-monomer [29]. In the XRD experiments, we obtained temperature dependence of $V^{\mathrm{Au}}(T)$ under the constant heating rate, $\Delta T / \Delta t(=10 \mathrm{~K} / \mathrm{min})$. If we assume that the growth curve, $V^{\mathrm{Au}}(T)$, has a similarity with time dependence of the volume, $V^{\mathrm{Au}}(t), \frac{\mathrm{d} V^{\mathrm{Au}}}{\mathrm{d} t} \cong \frac{\Delta V^{\mathrm{Au}}}{\Delta T} \frac{\Delta T}{\Delta t}$ is approximately obtained. With satisfying the diffusion-assisted nucleation, the Equation (3) is modified by,

$$
\frac{\Delta V^{\mathrm{Au}}}{\Delta T} \propto \frac{\mathrm{d} P}{\mathrm{~d} t} \propto r(T) D(T)\{c(T)\}^{2} \exp \left\{-\frac{\Delta G(r, T)}{k_{\mathrm{B}} T}\right\} .
$$

As mentioned earlier, Bragg intensity from c-Au (Figure 6(a)) is proportional to $V^{\mathrm{Au}}(T)$. The first derivative 
of the observed $V^{\mathrm{Au}}(T),\left(\mathrm{d} V^{\mathrm{Au}} / \mathrm{d} T\right)^{\text {obs }}$, is easily calculated. Also, $r^{\mathrm{obs}}(T)$ is estimated by FWHM of Bragg reflection (Figure 6(b)). We used $D(T)$ in the previous study [31]. Unknown parameters are $\Delta G$ and $c(T)$ in Equation (5). From 480 to $600 \mathrm{~K}$, we assume that $\Delta G$ in Equation (5) is approximately expressed by a weak linear relation with temperature ( $\Delta G=k_{0}+k_{1} T$ ). Also, it is assumed that $c(T)$ is described by proper function such as tanh $\left\{-k\left(T-T_{0}\right)\right\}$. By referring $\left(\mathrm{d} V^{\mathrm{Au}} / \mathrm{d} T\right)^{\text {obs }}$, the self-consistent calculation can determine the $\Delta G$ and $c(T)$ simultaneously. As a result, the calculated growth curve including diffusion process is exhibited by the solid curve in Figure 6(a). Above $560 \mathrm{~K}$, actual intensity of Au 111 Bragg reflection decreased due to melting. Finally, both experimental results and model calculation supports the idea based on the diffusion-assisted nucleation process of $\mathrm{Au}$. We notice that the initial state of the a-Au/a-Si thin film has major influence on both the irreversible MIC in $\mathrm{Si}$ and the Au nano-crystallization. In fact, the relatively slow diffusion of Au was enhanced in a-Si compared with Au diffusion in c-Si [31].

\subsection{Reversible Liquid-Au Nanoparticle Transition}

If irreversible morphological transformation from the multilayer to Au nanoparticle occurs, the reversible liquid-solid phase transition was observed repeatedly. Even above $T_{\mathrm{m}}^{\mathrm{NP}}$ of c-Au, the whole thin film does not melt, since c-Si and a-Si existed at $T_{\max }$ and the flat surface in the thin film was maintained at room temperature after thermal cycling. Heterogeneous $\ell$-AuSi in the thin film above $T_{\mathrm{m}}^{\mathrm{NP}}$ is the key to understand the reversible phase transition between the $\ell$-AuSi and the c-Au nanoparticles. On cooling at cycle $2, T_{\mathrm{c}}$ was determined to be $556 \mathrm{~K}$ by temperature dependences of intensity and FWHM of Au 111 Bragg reflection (Figure 7(a) and Figure 7(b)). Since small nuclei were not observable in small scattering volume of the thin film, Bragg intensities were derived not from small nuclei, but from the c-Au nanoparticles. A more significant point is that FWHM of the Bragg reflection is invariant on cooling process in Figure 7(b). It is well known that the constant FWHM of Bragg reflection means non-coarsening of a crystal domain [32]. Hence, the population of c-Au nanoparticles is increasing on cooling without crystal domain growth. The behavior is analogous with reversible spin ordering in a ferromagnet. Therefore, we apply the Ising model [33] into the crystallization from the $\ell$-AuSi to the c-Au nanoparticles. The feature of the Ising model is that the Hamiltonian is described only by the first nearest neighbor interaction. Based on the Ising model, crystallization process is provided by,

$$
V^{\mathrm{Au}}(T) \propto\left|T_{\mathrm{c}}-T\right|^{\beta},
$$

where $\beta$ is the critical exponent. By the least square fitting, the experimentally obtained $\beta^{\text {obs }}$ is evaluated to be 0.17. The fitted growth curve is represented by the solid line in Figure 6(a). The exact solution of the 2D Ising model showed the $\beta=1 / 8(=0.125)$ [33]. In order to investigate the crossover from 2D to 3D property in the Ising model, systematic simulations in the thin film [34] were demonstrated, where periodic boundary condition is employed on the $x-y$ planes. Here, the $x-y$ plane is parallel to the substrate and the $z$ direction is perpendicular to

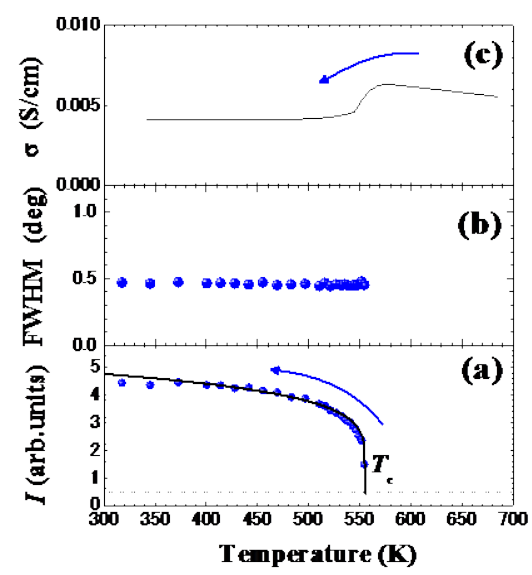

Figure 7. Cooling process of (a) intensity, (b) FWHM of Au 111 Bragg reflection at the 2nd cooling and (c) electrical conductivity, $\sigma$. Solid curve is obtained by the quasi 2D Ising model. 
it. In the simulation box denoted by $L \times L \times N_{\mathrm{z}}$, the critical exponent $\beta^{\text {cal }}$ becomes 0.17 , when $L$ is 160 and $N_{\mathrm{z}}$ is 6. The accordance between $\beta^{\text {obs }}$ and $\beta^{\text {cal }}$ indicates that the nanoparticles without their aggregations are consistent with the ideal spin system with finite thickness along the stacking sequence direction. Also, it is supposed that the actual appearance of the Au nanoparticles obeys short-range order (SRO) interaction. The analogy provides a significant insight into the reversible $\ell$-AuSi-c-Au nanoparticle phase transition. Figure 7(c) reveals electrical conductivity on cooling. Vanishing of $\ell$-AuSi causes the decrease of the conductivity at around $T_{\mathrm{c}}$. Below $T_{\mathrm{c}}$, the conductivity value became smaller than that on heating as shown in Figure 6(c), which is represented by the irreversible transformation from the multilayer to nano particle. At room temperature, the difference between them is originated from the layered a-Au land the isolated Au nanoparticles.

\subsection{Thermodynamic Analysis of Au Nanoparticle in Eutectic AuSi}

As mentioned earlier, the reversible liquid-solid phase transition in the thin film occurs between heterogeneous $\ell$-AuSi and c-Au nanoparticles. When we consider the surface and bulk phases at the equilibrium state, the surface tension in binary liquid alloy should be treated thermodynamically. Recently, it is predicted from a thermodynamical perspective, that spherical particle size [19] [35] is obtained by introducing the surface tension [36]. The relationships between the observed $T_{\mathrm{m}}^{\mathrm{NP}}$ and radius of the c-Au nanoparticles $\left(r^{\mathrm{obs}}\right)$ were calculated using the basic theory in nano-sized binary alloy system [19] [35]. In in situ XRD, $r^{\text {obs }}$ in cycle 2 - 4 is obtained by the Scherrer equation as listed in Table 2 [28]. While, $r^{\text {obs }}$ is also determined by direct TEM observations. In fact, the particle size of c-Au has a relation with $T_{\mathrm{m}}^{\mathrm{NP}}$ as shown in Figure 8.

In the theory [19] [35], the spherical particle having radius, $r$, is connected with $T_{\mathrm{m}}^{\mathrm{NP}}$ in binary alloy system. Gibbs energy of bulk ( $\Delta G^{\text {Bulk }}$ ) and surface ( $\Delta G^{\text {Surf }}$ ) in the binary system is given by,

$$
\begin{gathered}
\Delta G^{\text {Bulk }}=x_{1} \Delta G_{1}^{\mathrm{L}-\mathrm{S}}+x_{2} \Delta G_{2}^{\mathrm{L}-\mathrm{S}}+G^{\mathrm{ex}, \mathrm{L}}+R T\left(x_{1} \ln x_{1}+x_{2} \ln x_{2}\right), \\
\Delta G^{\mathrm{Surf}}=\frac{2}{r}\left\{\sigma^{\mathrm{L}} V^{\mathrm{L}}-\left(x_{1} \sigma_{1}^{\mathrm{S}} V_{1}^{\mathrm{S}}+x_{2} \sigma_{2}^{\mathrm{S}} V_{2}^{\mathrm{S}}\right)\right\} .
\end{gathered}
$$

Here, $\Delta G_{i}^{\mathrm{L}-\mathrm{S}}$ is Gibbs energy difference between liquid and solid in pure system (i-component: $\mathrm{i}=1$ or 2), while $G^{\mathrm{ex}, \mathrm{L}}$ is the excess Gibbs energy of liquid phase in binary system. Surface tension of pure solid is expressed by $\sigma_{\mathrm{i}}^{\mathrm{S}} \cdot \sigma^{\mathrm{L}}$ reveals the surface tension of binary liquid combined with molar volume of the binary liquid, $V^{\mathrm{L}} . \sigma^{\mathrm{L}}$ is numerically calculated using $\sigma_{1}^{\mathrm{L}}$ and $\sigma_{2}^{\mathrm{L}} \cdot V^{\mathrm{L}}$ is defined by $x_{1} V_{1}^{\mathrm{L}}+x_{2} V_{2}^{\mathrm{L}} \cdot V_{\mathrm{i}}^{\mathrm{L}}$ corresponds to the molar volume for the pure liquid component. In contrast, $\sigma_{\mathrm{i}}^{\mathrm{S}}$ and $V_{\mathrm{i}}^{\mathrm{S}}$ are surface tension and molar volume of pure solid, respectively. It should be noticed that $\sigma_{\mathrm{i}}^{\mathrm{L}}, V_{\mathrm{i}}^{\mathrm{L}}, \sigma_{\mathrm{i}}^{\mathrm{S}}$ and $V_{\mathrm{i}}^{\mathrm{S}}$ contain the temperature factor of $\left(T_{\mathrm{m}}^{\mathrm{NP}}-T_{\mathrm{m}, \mathrm{i}}\right) . T_{\mathrm{m}, \mathrm{i}}$ is the melting point in pure system i. If the condition of $\Delta G^{\text {Bulk }}+\Delta G^{\text {Surf }}=0$ is satisfied, Equation (8) is modified as,

$$
r=-\frac{2}{\Delta G^{\text {Bulk }}}\left\{\sigma^{\mathrm{L}} V^{\mathrm{L}}-\left(x_{1} \sigma_{1}^{\mathrm{S}} V_{1}^{\mathrm{S}}+x_{2} \sigma_{2}^{\mathrm{S}} V_{2}^{\mathrm{S}}\right)\right\} .
$$

Using the temperature factor and Equation (9), the $T_{\mathrm{m}}^{\mathrm{NP}}$ at fixing concentration $x_{1}\left(=1-x_{2}\right)$ is provided depending on the Au nanoparticle radius, $r$. At $x_{\mathrm{E}}=0.186$ in AuSi system, the calculated radius of the Au nanoparticle, $r^{\text {all }}$, is numerically obtained using each $T_{\mathrm{m}}^{\mathrm{NP}}$ (Table 2). Here, in the calculations, thermodynamic data and parameters in Au-Si system as previously reported paper [35] are used. Experimentally, $\ell$-AuSi, compositional fluctuations occur naturally. If we permit the possible compositional fluctuations, the $r^{\text {obs }}$ obtained both from XRD and TEM is in good agreement with the $r^{\text {cal }}$ within experimental errors (Figure 8). Consequently, in the thin film, the lowering eutectic point of the Au nanoparticle is well explained theoretically.

\subsection{Comparison between Irreversible and Reversible Transformations}

The scenario of two different irreversible/reversible transformations is illustrated in Figure 9. The irreversible transformation from the layered a-Au to c-Au nanoparticles is distinguished from the reversible liquid-nanocrystal transformation. MIC process of a-Si partially occurred from the as-deposited sample $\left(T>T_{\text {MIC }}^{\mathrm{ML}}\right)$. The partial crystallization of Si and the constant volume of Si over all thermal cycling reflect on little free energy difference between a-Si and c-Si in the specific thin film. The nucleation sites might be distorted positions in the vicinity of the $\mathrm{Al}_{2} \mathrm{O}_{3}$ substrate or the boundaries between a-Au and a-Si. The layered a-Au could be modified on 
Table 2. Thermal cycle dependence of melting point ( $\left.T_{\mathrm{m}}^{\mathrm{NP}}\right)$, calculated radius of Au nanoparticle $\left(r^{\mathrm{cal}}\right)$ and observed one $\left(r^{\text {obs }}\right)$ determined by XRD and TEM. $T_{\max }$ is the maximum temperature at each thermal cycles.

\begin{tabular}{cccccc}
\hline Cycle & $T_{\max }(\mathrm{K})$ & $T_{\mathrm{m}}^{\mathrm{NP}}(\mathrm{K})$ & $r^{\mathrm{cal}}(\mathrm{nm})$ & $r^{\mathrm{obs}}$ XRD $(\mathrm{nm})$ & $r^{\mathrm{obs}}$ TEM $(\mathrm{nm})$ \\
\hline 2nd & 673 & 600 & 2.2 & 3.2 & 3.0 \\
3rd & 873 & 612 & 3.6 & 3.8 & 5.0 \\
4th & 1073 & 621 & 6.3 & 4.0 & 7.5 \\
\hline
\end{tabular}

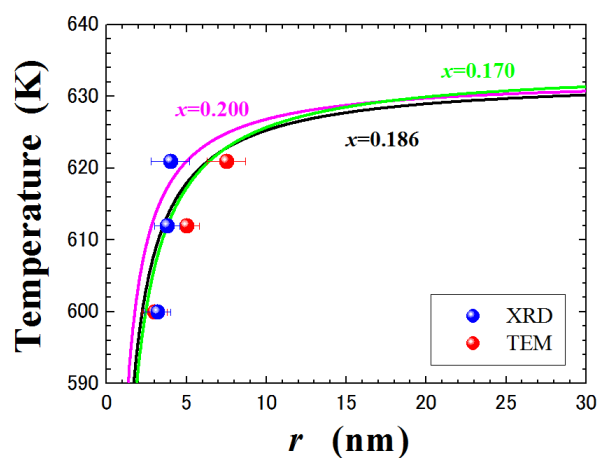

Figure 8. Thermodynamically calculated melting point in $\mathrm{Au}_{1-x} \mathrm{Si}_{x}$. Closed red and blue circles reveal the experimentally obtained radius by TEM and XRD.

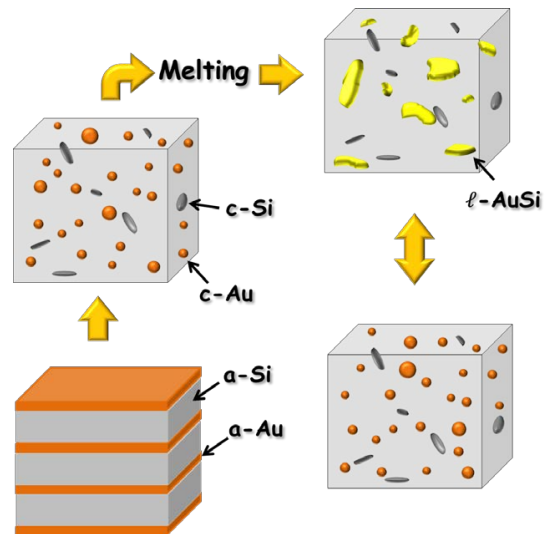

Figure 9. Schematic illustration of morphological transformation from a-Au/Si multilayer to Au nanoparticles.

atomic scale by crystal nucleation of Si, however, crystal nucleation of Au is not induced at this stage.

Next step on heating is that zigzag structure and Au crystallization occurs simultaneously as a precursor phenomenon of Au nanoparticle formation ( $T>T_{\mathrm{c}}-T_{\mathrm{zig}}$ ). As a result of aggregation of diffusing Au atoms, Au nucleation takes place on the off-sites from the original a-Au layers. Remarkably slow temperature evolution of $V^{\mathrm{Au}}(T)$ has no discrepancy with time consuming diffusion-driven nucleation. At the same time, shape transfer based on the diffusion-limited model happens in low energy barrier. When the interface-limited process occurs in the morphological transformation, large strain and boundary energy should be considered additionally. In spite of the diffusion-limited motion, all of a-Au is crystallized due to large driving force between amorphous and crystal.

Focusing on the reversible transformation, heterogeneous distribution of a-Si $\left(V_{\mathrm{Si}}-66.8 \%\right)$ at $T_{\max }$ prevents to melt the whole film $\left(T>T_{\mathrm{m}}\right)$. Partially melted $\ell$-AuSi region distributes independently in the center of c-Au before melting. On cooling, crystal nuclei of Au appear inside the isolated regions. With excluding Si atoms, the nuclei develop to the c-Au nanoparticles once those exceed over the critical size. Inside the $\ell$-AuSi, the nanoparticle size of c-Au is governed by the surface tension. The size of c-Au is optimized by a competition between bulk and surface free energy. The space restriction of the confined $\ell$-AuSi avoids aggregation between the c-Au 
nanoparticles. Then, the same reversible phase transition between the $\ell$-AuSi and the c-Au nanoparticles occurs repeatedly. Also, less degrees of freedom in the crystallization mechanism leads to the ideal Ising model described only by simple SRO correlation. The idealized spin system is applied into the spontaneous nucleation and size-limited growth in the thin film.

\section{Conclusions}

On heating, Au nanoparticle formation process from multilayer was clarified in situ TEM and XRD. The direct observation proves irreversibly morphological transformation from the layered a-Au/a-Si multilayer to the c-Au nanoparticles. As a precursor phenomenon to the Au nanoparticles, the intermediate zigzag patterns in real space were observed by in situ TEM. The zigzag structure was induced by diffusion-assisted Au crystallization. While, reversible transformation between the $\ell$-AuSi to the c-Au nanoparticles was obtained by in situ XRD. The reversible phenomenon is a typical memory effect in the thin film. Heterogeneous eutectic $\ell$-AuSi surrounding by deformed a-Si matrix contributes to reproducible appearance of c-Au nanoparticles, whose position and size are almost fixed. $V^{\mathrm{Au}}(T)$ as the outstanding memory effect is well explained by the quasi-2D Ising model.

Theoretically, the relation between $T_{\mathrm{m}}^{\mathrm{NP}}$ and the particle size can support the experimental results in the eutectic AuSi binary system. The size of c-Au from the $\ell$-AuSi is proportional to the driving force depending on $T_{\max }$ at each thermal cycle.

\section{Acknowledgements}

We appreciate Dr. Y. Okamoto and Professor Y. Watanabe of National Defense Academy for sample preparations and helpful discussions. This work was supported by NIMS (National Institute for Materials Science, Japan) microstructural characterization platform as a program of "Nanotechnology Platform" of the Ministry of Education, Culture, Sports, Science and Technology (MEXT), Japan.

\section{References}

[1] Hiraki, A. (1983) Low Temperature Reactions at Si-Metal Contacts—From $\mathrm{SiO}_{2}$ Growth Due to Si-Au Reaction to the Mechanism of Silicide Formation. Japanese Journal of Applied Physics, 22, 549-562. http://dx.doi.org/10.1143/JJAP.22.549

[2] Ito, T. and Gibson, W.M. (1987) Metal/Silicon Reactions Studied by High Energy Ion Scattering. Japanese Journal of Applied Physics, 26, 841-847. http://dx.doi.org/10.1143/JJAP.26.841

[3] Her, Y.-C. and Chen, C.-W. (2007) Crystallization Kinetics of Ultrathin Amorphous Si Film Induced by Al Metal Layer under Thermal Annealing and Pulsed Laser Irradiation. Journal of Applied Physics, 101, Article ID: 043518. http://dx.doi.org/10.1063/1.2654512

[4] Bokhonov, B. and Korchagin, M. (2000) In Situ Investigation of Stage of the Formation of Eutectic Alloys in Si-Au and Si-Al Systems. Journal of Alloys and Compounds, 312, 238-250. http://dx.doi.org/10.1016/S0925-8388(00)01173-7

[5] Ehrhardt, J., Klimmer, A., Eisenmenger, J., Müller, Th., Boyen, H.-G., Ziemanna, P., Biskupek, J. and Kaiser, U. (2006) Influence of Ion Induced Amorphicity on the Diffusion of Gold into Silicon. Journal of Applied Physics, 100, Article ID: 063534. http://dx.doi.org/10.1063/1.2259815

[6] Knaepen, W., Detavernier, C., Van Meirhaeghe, R.L., Sweet, J.J. and Lavoie, C. (2008) In-Situ X-Ray Diffraction Study of Metal Induced Crystallization of Amorphous Silicon. Thin Solid Films, 516, 4946-4952. http://dx.doi.org/10.1016/j.tsf.2007.09.037

[7] Bal, J.K. and Hazra, S. (2007) Interfacial Role in Room-Temperature Diffusion of Au into Si Substrates. Physical Review B, 75, Article ID: 205411. http://dx.doi.org/10.1103/PhysRevB.75.205411

[8] Ashtikar, M.S. and Sharma, G.L. (1995) Structural Investigation of Gold Induced Crystallization in Hydrogenated Amorphous Silicon Thin Films. Japanese Journal of Applied Physics, 34, 5520-5526. http://dx.doi.org/10.1143/JJAP.34.5520

[9] Chromik, R.R., Zavalij, L., Johnson, M.D. and Cotts, E.J. (2002) Calorimetric Investigation of the Formation of Metastable Silicides in Au/a-Si Thin Film Multilayers. Journal of Applied Physics, 91, 8992-8998. http://dx.doi.org/10.1063/1.1432774

[10] Wu, J.S., Dhara, S., Wu, C.T., Chen, K.H., Chen, Y.F. and Chen, L.C. (2002) Growth and Optical Properties of SelfOrganized $\mathrm{Au}_{2} \mathrm{Si}$ Nanospheres Pea-Podded in a Silicon Oxide Nanowire. Advanced Materials, 14, 1847-1850. 
http://dx.doi.org/10.1002/adma.200290017

[11] Shpyrko, O.G., Streitel, R., Balagurusamy, V.S.K., Grigoriev, A.Y., Deutsch, M., Ocko, B.M., Meron, M., Lin, B. and Pershan, P.S. (2007) Crystalline Surface Phases of the Liquid Au-Si Eutectic Alloy. Physical Review B, 76, Article ID: 245436. http://dx.doi.org/10.1103/PhysRevB.76.245436

[12] Pinardi, A.L., Leake, S.J., Felici, R. and Robinson, I.K. (2009) Formation of an Au-Si Eutectic on a Clean Silicon Surface. Physical Review B, 79, Article ID: 045416. http://dx.doi.org/10.1103/PhysRevB.79.045416

[13] Schülli, T.U., Daudin, R., Renaud, G., Vaysset, A., Geaymond, O. and Pasturel, A. (2010) Substrate-Enhanced Supercooling in AuSi Eutectic Droplets. Nature, 464, 1174-1177. http://dx.doi.org/10.1038/nature08986

[14] Chandra, A. and Clemens, B.M.J. (2004) Monodisperse Nanoparticles via Metal Induced Crystallization. Journal of Applied Physics, 96, 6776-6781. http://dx.doi.org/10.1063/1.1812817

[15] Venkatachalam, D.K., Fletcher, N.H., Sood, D.K. and Elliman, R.G. (2009) Self-Assembled Nanoparticle Spirals from Two-Dimensional Compositional Banding in Thin Films. Applied Physics Letters, 94, Article ID: 213110. http://dx.doi.org/10.1063/1.3143666

[16] Aono, M., Takahashi, M., Takiguchi, H., Okamoto, Y., Kitazawa, N. and Watanabe, Y. (2012) Thermal Annealing of a-Si/Au Superlattice Thin Films. Journal of Non-Crystalline Solids, 358, 2150-2153. http://dx.doi.org/10.1016/j.jnoncrysol.2011.12.088

[17] Miyazaki, H., Takiguchi, H., Aono, M. and Okamoto, Y. (2012) Influence of Annealing Temperature and Au Concentration on the Electrical Properties of Multilayered a-Ge/Au Films. Journal of Non-Crystalline Solids, 358, 2103-2106. http://dx.doi.org/10.1016/j.jnoncrysol.2012.01.064

[18] Stockman, M.I. (2010) Nanoscience: Dark-Hot Resonances. Nature, 467, 541-542.

[19] Derkacs, D., Lim, S.H., Matheu, P., Mar, W. and Yu, E.T. (2006) Improved Performance of Amorphous Silicon Solar Cells via Scattering from Surface Plasmon Polaritons in Nearby Metallic Nanoparticles. Applied Physics Letters, 89, Article ID: 093103. http://dx.doi.org/10.1063/1.2336629

[20] Fujiki, A., Uemura, T., Zettsu, N., Akai-Kasaya, M., Saito, A. and Kuwahara, Y. (2010) Enhanced Fluorescence by Surface Plasmon Coupling of Au Nanoparticles in an Organic Electroluminescence Diode. Applied Physics Letters, 96, Article ID: 043307. http://dx.doi.org/10.1063/1.3271773

[21] Cheng, C.W., Sie, E.J., Liu, B., Huan, C.H.A., Sum, T.C., Sun, H.D. and Fan, H.J. (2010) Surface Plasmon Enhanced Band Edge Luminescence of ZnO Nanorods by Capping Au Nanoparticles. Applied Physics Letters, 96, Article ID: 071107. http://dx.doi.org/10.1063/1.3323091

[22] Wu, T.H., Kuo, P.C., Ou, S.L., Chen, J.P., Yen, P.F., Jeng, T.R., Wu, C.Y. and Huang, D.R. (2008) Diffusion and Crystallization Mechanisms of Ge/Au Bilayer Media for Write-Once Optical Disk. Applied Physics Letters, 92, Article ID: 011126. http://dx.doi.org/10.1063/1.2831690

[23] Guzman, J., Boswell-Koller, C.N., Beeman, J.W., Bustillo, K.C., Conry, T., Dubón, O.D., Hansen, W.L., Levander, A. X., Liao, C.Y., Lieten, R.R., Sawyer, C.A., Sherburne, M.P., Shin, S.J., Stone, P.R., Watanabe, M., Yu, K.M., Ager III, J.W., Chrzan, D.C. and Haller, E.E. (2011) Reversible Phase Changes in Ge-Au Nanoparticles. Applied Physics Letters, 98, Article ID: 193101. http://dx.doi.org/10.1063/1.3584850

[24] Siegel, J., Lyutakov, O., Rybka, V., Kolská, Z. and Švorčík, V. (2011) Properties of Gold Nanostructures Sputtered on Glass. Nanoscale Research Letters, 6, 96-99. http://dx.doi.org/10.1186/1556-276X-6-96

[25] Alvarez, F., Díaz, C.C., Valladares, A.A. and Valladares, R.M. (2002) Radial Distribution Functions of $a b$ Initio Generated Amorphous Covalent Networks. Physical Review B, 65, Article ID: 113108 http://dx.doi.org/10.1103/PhysRevB.65.113108

[26] Mittemeijer, E.J., Welzel, U. and Kristallogr, Z. (2008) The "State of the Art" of the Diffraction Analysis of Crystallite Size and Lattice Strain. Zeitschrift für Kristallographie, Crystalline Materials, 223, 552-560. http://dx.doi.org/10.1524/zkri.2008.1213

[27] Warren, B.E. (1990) X-Ray Diffraction. Dover, New York.

[28] Ida, T., Shimazaki, S., Hibino, H. and Toraya, H. (2003) Diffraction Peak Profiles from Spherical Crystallites with Lognormal Size Distribution. Journal of Applied Crystallography, 36, 1107-1115. http://dx.doi.org/10.1107/S0021889803011580

[29] Robb, D.T. and Privman, V. (2008) Model of Nanocrystal Formation in Solution by Burst Nucleation and Diffusional Growth. Langmuir, 24, 26-35. http://dx.doi.org/10.1021/la702097g

[30] Privman, V. (2008) Diffusional Nucleation of Nanocrystals and Their Self-Assembly into Uniform Colloids. Journal of Optoelectronics and Advanced Materials, 10, 2827-2839.

[31] Nygren, E., Park, B., Goldman, L.M. and Spaepen, F. (1990) Diffusivity of Gold in Amorphous Silicon Measured by the Artificial Multilayer Technique. Applied Physics Letters, 56, 2094-2096. http://dx.doi.org/10.1063/1.102983 
[32] Abe, H., Ishibashi, M., Ohshima, K., Suzuki, T., Wuttig, M. and Kakurai, K. (1994) Kinetics of the Martensitic Transition in In-Tl Alloys. Physical Review B, 50, 9020-9024. http://dx.doi.org/10.1103/PhysRevB.50.9020

[33] Onsager, L. (1944) Crystal Statistics. I. A Two-Dimensional Model with an Order-Disorder Transition. Physical Review, 65, 117-149. http://dx.doi.org/10.1103/PhysRev.65.117

[34] Phu, X.T.P., Ngo, V.T. and Diep, H.T. (2009) Critical Behavior of Magnetic Thin Films. Surface Science, 603, 109116. http://dx.doi.org/10.1016/j.susc.2008.10.037

[35] Tanaka, T. (2010) Prediction of Phase Diagrams in Nano-Sized Binary Alloys. Materials Science Forum, 653, 55-75. http://dx.doi.org/10.4028/www.scientific.net/MSF.653.55

[36] Yeum, K.S., Speiser, R. and Poirier, D.R. (1989) Estimation of the Surface Tensions of Binary Liquid Alloys. Metallurgical Transactions B, 20, 693-703. 
Scientific Research Publishing (SCIRP) is one of the largest Open Access journal publishers. It is currently publishing more than 200 open access, online, peer-reviewed journals covering a wide range of academic disciplines. SCIRP serves the worldwide academic communities and contributes to the progress and application of science with its publication.

Other selected journals from SCIRP are listed as below. Submit your manuscript to us via either submit@scirp.org or Online Submission Portal.
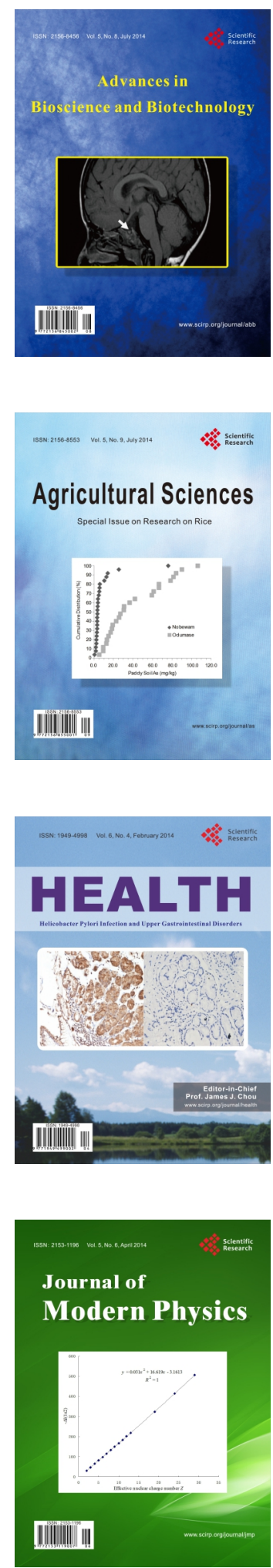
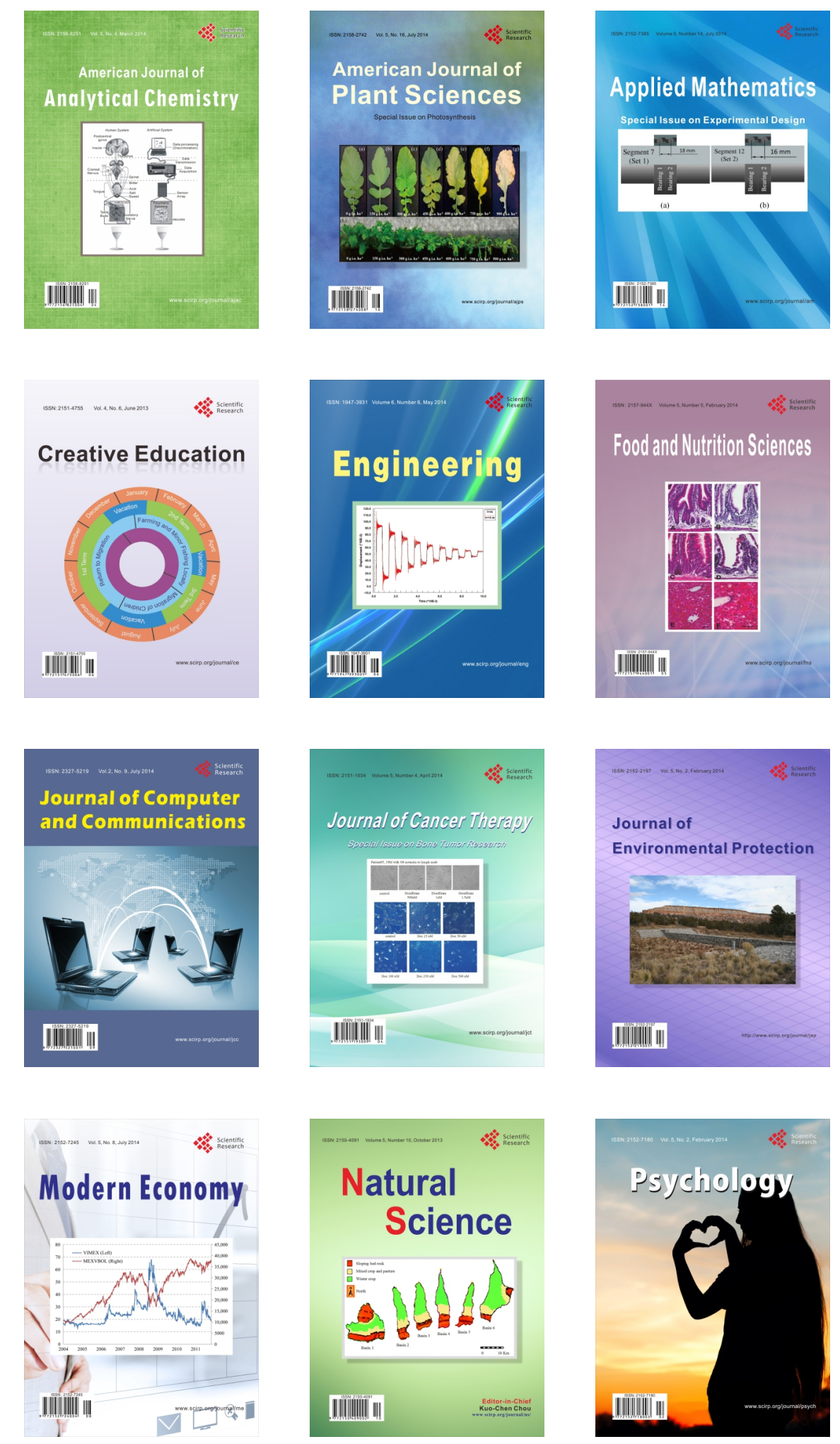